\title{
Cell sheet technology-driven re-epithelialization and neovascularization of skin wounds
}

\author{
M.T. Cerqueira, R.P. Pirraco, A.R. Martins, T.C. Santos, R.L. Reis, A.P. Marques* \\ 3B's Research Group - Biomaterials, Biodegradables and Biomimetics, University of Minho, Headquarters of the European Institute of Excellence on Tissue Engineering \\ and Regenerative Medicine, AvePark, Zona Industrial da Gandra, S. Cláudio do Barco, 4806-909 Taipas, Guimarães, Portugal \\ ICVS/3B's - PT Government Associate Laboratory, Braga, Guimarães, Portugal
}

\section{A R T I C L E I N F O}

\section{Article history:}

Received 1 August 2013

Received in revised form 12 February 2014

Accepted 9 March 2014

Available online 18 March 2014

\section{Keywords:}

Cell sheet engineering

Skin lineages

Skin regeneration

Wound healing

\begin{abstract}
A B S T R A C T
Skin regeneration remains a challenge, requiring a well-orchestrated interplay of cell-cell and cellmatrix signalling. Cell sheet (CS) engineering, which has the major advantage of allowing the retrieval of the intact cell layers along with their naturally organized extracellular matrix (ECM), has been poorly explored for the purpose of creating skin substitutes and skin regeneration. This work proposes the use of CS technology to engineer cellular constructs based on human keratinocytes (hKC), key players in wound re-epithelialization, dermal fibroblasts ( $\mathrm{hDFb}$ ), responsible for ECM remodelling, and dermal microvascular endothelial cells (hDMEC), part of the dermal vascular network and modulators of angiogenesis. Homotypic and heterotypic three-dimensional (3-D) CS-based constructs were developed simultaneously to target wound re-vascularization and re-epithelialization. After implantation of the constructs in murine full-thickness wounds, human cells were engrafted into the host wound bed and were present in the neotissue formed up to 14 days post-implantation. Different outcomes were obtained by varying the composition and organization of the 3-D constructs. Both hKC and hDMEC significantly contributed to re-epithelialization by promoting rapid wound closure and early epithelial coverage. Moreover, a significant increase in the density of vessels at day 7 and the incorporation of hDMEC in the neoformed vasculature confirmed its role over neotissue vacularization. As a whole, the obtained results confirmed that the proposed 3-D CS-based constructs provided the necessary cell machinery, when in a specific microenvironment, guiding both re-vascularization and re-epithelialization. Although dependent on the nature of the constructs, the results obtained sustain the hypothesis that different CS-based constructs lead to improved skin healing.
\end{abstract}

(c) 2014 Acta Materialia Inc. Published by Elsevier Ltd. All rights reserved.

\section{Introduction}

Autologous and allogeneic traditional epidermal sheets, available for nearly three decades, have proved to be successful in the re-epithelialization of split-thickness wounds [1-10]. It is well known that these cells secrete a multitude of biologically relevant molecules that contribute positively to wound healing [11,12]. However, various difficulties and drawbacks have been encountered in the use of these sheets, which have significantly compromised their clinical application and outcomes. Among those, the 23 week autograft production period [2] severely compromises patients' recovery, impairing its application, for example, in the treatment of extensive skin lesions. In addition to this, the instability and the variable wound residence time $[10,13]$, as well as failure

\footnotetext{
* Corresponding author. Tel.: +351510906.

E-mail address: apmarques@dep.uminho.pt (A.P. Marques).
}

to prevent wound contraction [13] are also limiting in traditional epidermal sheets. Epidermal sheets from patients' own hair follicles, epidex, have been proposed recently [14], but the availability of the grafts in clinically relevant sizes is still an important issue. Some studies have also highlighted the limited action of keratinocytes in the formation of granulation tissue or dermal remodelling in full-thickness wounds $[15,16]$, and in the healing of full-thickness burns in the absence of a well-vascularized dermal wound bed [17], reinforcing the importance of a dermal component in skin tissue engineering (TE) constructs.

Fibroblast sheets have acted as dermal elements in different skin TE approaches; the first followed a two-step methodology in which a keratinocyte sheet is transplanted onto full-thickness wounds previously grafted with a fibroblast sheet $[18,19]$. However, the fragility of the sheet's extracellular matrix (ECM) and the delayed re-epithelialization associated with the two-step implantation still represent significant limitations. Bi-layered skin 
analogues with a dermal and epidermal part, formed by assembling superimposed mature fibroblasts sheets with keratinocytes cultured on top, were developed to tackle those issues [20]. A step forward was taken with the creation of endothelialized grafts, aiming at a fast and efficient re-establishment of capillary flow at an early stage of implantation. In a recently explored approach using stacked cell sheets (CS) of fibroblasts, endothelial cells and keratinocytes obtained under different culture conditions, the importance of the co-culture of different cell components and its effect on angiogenic factor secretion was stressed [21]. Moreover, endothelial cells have been co-cultured with fibroblasts, forming heterotypic sheets aiming to improve construct vascularization $[21,22]$. Despite the exciting results obtained with these methodologies, the results refer to in vitro performance. Moreover, the production of a consistent bilayer structure requires a significantly wide time-frame to include the time needed from the isolation of the different cell types to the consequent maturation steps prior to implantation of the assembled construct, which could compromise the time window in a future clinical setting.

From a similar perspective, CS engineering in thermo-responsive surfaces has been proposed to obtain scaffold-free TE constructs. The value of this technology for treating cornea defects [23], cardiac problems [24] and for periodontal ligament [25] and bladder regeneration [26] has been well demonstrated. Nonetheless, the use of CS engineering technology in the creation of strategies for skin TE has just started to be explored. To the best of the present authors' knowledge, it is limited to the in vitro generation of keratinocytes CS, avoiding the use of dispase and allowing the production of easily handled sheets [27], and more recently to the creation of stem-cell-based constructs for the regeneration of full-thickness skin wounds $[28,29]$. A major advantage of CS engineering is the use of thermo-responsive surfaces that allow CS retrieval by temperature decrease with the ECM intact [30]. The naturally adhesive character of this ECM promotes CS re-attachment to other surfaces or tissues [31], as well the assembling of three-dimensional (3-D) constructs of layered CS [30,32-39], thus representing a major benefit. In fact, increased cellular residence time on transplantation has been observed in studies using constructs created by CS engineering [29,40,41]. In this sense, using this technology to facilitate wound healing, potentially promoting skin regeneration of functional skin, can present several advantages worth exploring.

This particular work adds to the traditional sheet grafting approach the possibility of generating confluent and easily retrieved CS in a shorter time-frame, as previously optimized by the present authors for other cell types [29], and the subsequent creation of stable constructs, as a result of the particular ECM adhesive character, easily transplanted into the wound site without an intermediate maturation step. Cell isolation and expansion constraints, particularly in the case of keratinocytes [42] and endothelial cells [43], are also tackled in the approach proposed herein by the use of hDFb in all the constructs, which produce an ECM-rich template to co-culture hKC and hDMEC, thus avoiding prolonged culture times. Thus, this study aims to take advantage of the unique features of CS engineering technology to engineer relevant cellularbased constructs for skin regeneration. In particular, the present authors propose the use of human keratinocytes (hKC), which are key players in wound re-epithelialization [44], dermal fibroblasts (hDFb), mainly responsible for ECM remodelling [45], and dermal microvascular endothelial cells (hDMEC), which are part of the dermal vascular network and modulators of angiogenic signalling [46], to design homotypic and heterotypic CS. These cell types were then used to assemble different CS-based constructs with distinct designs expected to provide the necessary cell machinery within a specific microenvironment defined by their native ECM, and to attain cellular-based constructs capable of leading specific wound-healing stages, such as re-vascularization and re-epithelialization. Two CS of hDFb co-cultured with hDMEC were stacked in order to assemble a construct where the hDMEC were both inbetween the hDFb and on one side of the construct, for direct interaction with the wound bed after transplantation, hypothesized as a way of promoting faster inosculation. A second construct combined a homotypic hDFb CS with a second CS of hDFb co-cultured with hKC, located in the outer layer of the construct, hypothesized as a way to contribute to the re-epithelialization of the wound. The third construct was assembled with two heterotypic CS: a CS of $\mathrm{hDFb}$ co-cultured with $\mathrm{hKC}$, at the most exterior layer of the construct, combined with a CS of hDFb co-cultured with hDMEC, located between the hDFb of the two CS, to target both reepithelialization and neovascularization. Overall, it is hypothesized that both the nature and design of engineered constructs created through CS technology distinctively affect the progression of wound healing, namely re-epithelialization and re-vascularization.

\section{Materials and methods}

\subsection{Skin cells isolation and culture}

Human KC, DMEC and DFb were harvested from human skin samples obtained from abdominoplasties performed at Hospital da Prelada (Porto, Portugal), after informed consent. Samples were obtained under a collaboration protocol with the 3B's Research Group, approved by the ethical committees of both institutions. Briefly, skin specimens were cut into small fragments and incubated overnight in dispase $\left(2.4 \mu \mathrm{ml}^{-1}\right)$ (BD Biosciences, USA) at $4{ }^{\circ} \mathrm{C}$. Epidermis was then peeled off and digested with $0.05 \%$ Trypsin-EDTA (Invitrogen, USA) at $37^{\circ} \mathrm{C}$. The digested tissue was filtered using a sterile $100 \mu \mathrm{m}$ cell strainer (BD Biosciences, USA) and centrifuged, and the isolated hKC were cultured in keratinocyte serum-free medium (KSFM) (Invitrogen, USA) with $1 \%$ antibiotic/antimycotic (Invitrogen, USA). The medium was changed every 3 days. On dissociation from the epidermis, the dermis was further digested in $0.1 \%$ collagenase type I (Sigma, USA) for $3 \mathrm{~h}$ at $37^{\circ} \mathrm{C}$, under agitation. Again, digested tissue was passed through a $100 \mu \mathrm{m}$ cell strainer, and the hDFb obtained were cultured in a-MEM medium (Invitrogen, USA) supplemented with $10 \%$ fetal bovine serum (FBS) (Invitrogen, USA) and 1\% antibiotic/antimycotic. hDMEC were obtained through the filtration and centrifugation of the discarded dispase solution, after dermis mechanical dissociation. hDMEC were cultured in $0.7 \%$ gelatin (Sigma, USA) coated flasks with endothelial growth medium (EGM2, Lonza, USA). hDFb and hDMEC were used at passage $3-4$, while hKC were used at passage 1 .

\subsection{CS fabrication}

The hDFb at a density of $5 \times 10^{4}$ cells $\mathrm{cm}^{-2}$ were plated in commercially available UP cell $35 \mathrm{~mm}$ thermo-responsive dishes (Nunc, Thermo Scientific, Denmark) and cultured for 5 days in alpha-MEM medium, supplemented with $10 \% \mathrm{FBS}, 1 \% \mathrm{AB}$ and $50 \mu \mathrm{g} \mathrm{ml}^{-1}$ of ascorbic acid (Sigma, Germany) at $37^{\circ} \mathrm{C}$ in a humidified atmosphere with $5 \% \mathrm{CO}_{2}$ [29]. Heterotypic CS were fabricated, combining a monolayer of hDFb with either hDMEC or hKC cultured on top. In more detail, at day $3,5 \times 10^{5}$ hDMEC or $8 \times 10^{6}$ $\mathrm{hKC}$ were seeded on top of the hDFb monolayer and co-cultured in EGM-2MV and in a mixture of KSFM/ $\alpha$-MEM medium $(1: 1)$, respectively, for a further 2 days. The same conditions were replicated in tissue culture polystyrene (TCPS) coverslips (Sarstedt, USA) for characterization without cell detachment. 


\subsection{CS stacking and construct preparation}

At day 5 of culture, constructs were prepared by CS stacking, based on the methodology described by Haraguchi et al. [31], with some modifications. Briefly, after removing the culture medium, a poly(vinylidene difluoride) (Immobilon-P, DURAPORE; Millipore Corporation, USA) membrane was placed over the cells and incubated at room temperature for $10 \mathrm{~min}$ for CS detachment. The culture medium from a second thermo-responsive dish was aspirated, and the first CS, spontaneously detached, was placed on the top of the second. The construct was incubated for $20 \mathrm{~min}$ at $37^{\circ} \mathrm{C}$ to promote CS adherence, generating a two-CS-stacked construct. In order to confirm the robustness of the retrieved CS by temperature reduction, a video showing the retrieval of the hDFb CS from the thermo-responsive dishes and respective manipulation is provided (Supplementary Material).

Three different constructs were created (Fig. 1): (i) two CS of hDFb co-cultured with hDMEC (hDFb + hDMEC); (ii) hDFb CS combined with a CS of hDFb co-cultured with hKC (hDFb + hKC); (iii) CS of $\mathrm{hDFb}$ co-cultured with hDMEC plus a second CS of hDFb co-cultured with hKC (hDFb + hDMEC + hKC).

\subsection{Transplantation into full-thickness excisional wound model in mice}

Sixty Swiss $\mathrm{Nu} / \mathrm{Nu}$ male mice (Charles River Laboratories, France), after approval by the Direcção Geral de Alimentação e Veterinária, the Portuguese National Authority for Animal Health, were randomly divided into three groups corresponding to three types of CS-based constructs, and the control group. Four animals were used per condition and per time point (3, 7, 14 and 21 days). Mice were anaesthetized with a mixture of Imalgene (ketamine) ( $75 \mathrm{mg} \mathrm{kg}^{-1}$ ) (Merial Portuguesa, Portugal) plus Domitor (medetomidine) $\left(1 \mathrm{mg} \mathrm{kg}^{-1}\right)$ (Esteve Farma, LDA, Portugal). A 1-cm-diameter full-thickness excision was performed on each mouse $\sim 0.5 \mathrm{~cm}$ caudal to the intra-scapular region. After transplantation, wounds were covered with Hydrofilm transparent dressing (Hartmann, UK), and a final set of bandages was used in order to avoid the dislocation of the transparent dressing and to protect the whole treatment set. Control wounds were left empty and dressed similarly. A subcutaneous injection of Depomedrol
(20 $\mathrm{mg} \mathrm{kg}^{-1}$ BW) (Pfizer, Portugal) was applied to the animals at days 0,7 and 14, in order to delay wound healing [47]. The animals were kept separately and, at the established endpoints, were euthanized by $\mathrm{CO}_{2}$ inhalation, and the explants were processed for histological analysis. All the surgical and necropsy procedures were performed according to the applicable national regulations respecting international animal welfare rules.

\subsection{Histological analysis}

The CS-based constructs created in vitro and the in vivo explants were fixed overnight in 3.7\% (v/v) buffered formalin (Sigma, USA), paraffin embedded and sectioned for posterior staining and analysis. All samples were analysed using an Axioplan Imager Z1 m microscope (Zeiss, Germany), and images were acquired and processed with AxioVision V.4.8 software (Zeiss, Germany).

\subsubsection{Immunostaining}

Immunolabelling of the CS in the TCPS prior to detachment was performed after fixation with $3.7 \%(\mathrm{v} / \mathrm{v})$ buffered formalin, permeabilization with $0.2 \%$ TritonX-100 blocking with $3 \%$ bovine serum albumin (BSA) (Sigma). The primary antibodies, CD31 (mouse anti-human, 1:30, Dako, Denmark), von Willebrand factor (vWF) (rabbit anti-human/mouse, 1:200, Dako, Denmark), fibroblast surface protein (FSP) (mouse anti-human, 1:80, Sigma, USA) and keratin 14 (K14) (rabbit anti-human/mouse, 1:100, Covance, USA), and the tagged secondary antibodies, rabbit anti-mouse Alexafluor 594 and donkey anti-rabbit Alexafluor 488 (1:500, invitrogen, USA), were used. Cell nuclei were stained with DAPI (Invitrogen, USA). Co-cultured hDMEC in the heterotypic CS were identified by Dil-AC-LDL uptake.

For paraffin embedded samples, sections were re-hydrated and incubated with antigen retrieval solution (Tris-EDTA buffer: $10 \mathrm{mM}$ Tris base, $1 \mathrm{mM}$ EDTA solution, 0.05\% Tween 20, pH 9.0) (all from Sigma, USA). Permeabilization was performed with $0.2 \%$ TritonX-100, followed by blocking with 3\% BSA (Sigma). Primary antibodies against keratin 14 (rabbit-anti-human/mouse, 1:800, Covance, USA), CD31 (human/mouse, 1:20, Abcam, UK), vWF and FSP were used. Alexafluor 488 donkey anti-rabbit and Alexafluor 594 rabbit anti-mouse were used as secondary antibodies. Histological samples of day 3 in vivo explants were incubated with

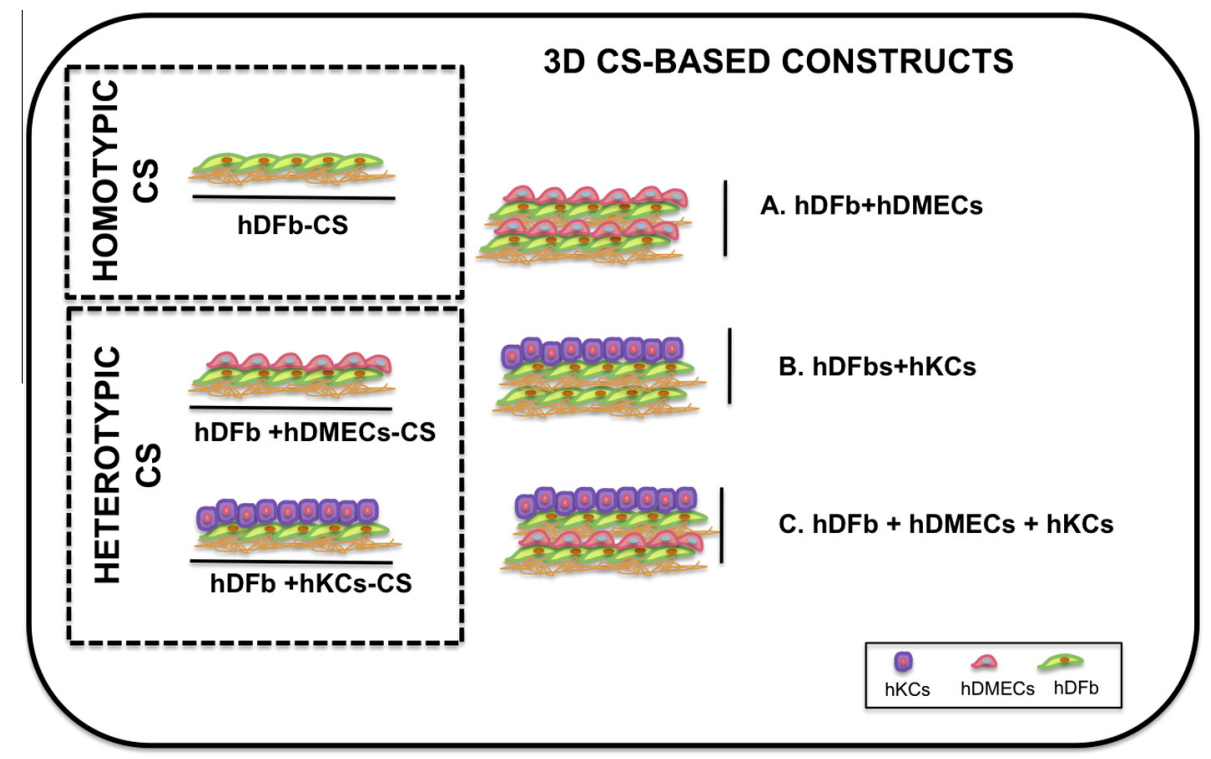

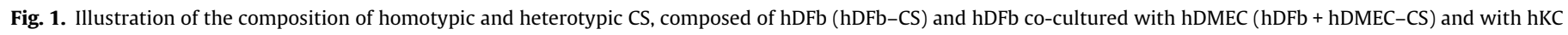
(hDFbs + hKC-CS) and the respective combinations generating distinct 3-D CS-based constructs. 
lectin from Ulex Europaeus Fluorescein (Sigma, USA) 1:100 in PBS for $1 \mathrm{~h}$ at $37^{\circ} \mathrm{C}$, and nuclei were counterstained with DAPI. For CD31 detection, VECTASTAIN Elite ABC Kit (Vector Labs, USA) was used, according to the manufacturer's instructions. Nuclei were counterstained with Mayer's haematoxylin.

\subsubsection{Chromogenic in situ hybridization (CISH)}

Histological samples of in vivo explants were deparaffinized, dehydrated, air dried, heated in a water bath at $95{ }^{\circ} \mathrm{C}$ in pre-treatment $1 \%$ MES buffer (Sigma, USA) for $10 \mathrm{~min}$ and digested with pepsin $\left(4 \mathrm{mg} \mathrm{ml}^{-1}\right)$ (Worthington, USA). After dehydration, histological slides were air-dried and the positive DNA-biotin labelled probe (Pan Path, Netherlands) was applied. Doublestranded DNA denaturation was performed by incubating the slides on a $95{ }^{\circ} \mathrm{C}$ heating block for $5 \mathrm{~min}$, and hybridization was achieved at $37^{\circ} \mathrm{C}$ overnight. Slides were then washed with PBS and with a stringency wash buffer (Pan Path, Netherlands) at $37^{\circ} \mathrm{C}$. For detection, streptavidin-peroxidase complex (Vector Labs, USA) was added for 20 min at RT. Revelation was completed with a DAB chromogen substrate system (Vector Labs, USA). Mayer's haematoxylin was used for counterstaining.

\subsection{Image analysis}

Analysis for the quantification of wound closure, vessel density and diameter, and epidermal thickness was carried out on explant images obtained using Image J software (Wayne, Rasband, NIH, USA), after processing and under the conditions detailed in each of the sections below.

\subsubsection{Wound closure}

Planimetric digital images were taken on the day of surgery and at days, 7, 14 and 21 post-surgery for all four animals per condition and time point. The percentage of wound closure was then calculated as

$\%$ of wound closure $=\frac{\text { area of original wound }- \text { area of actual wound }}{\text { area of original wound }}$ $\times 100$

A wound was considered completely closed when the wound area was equal to zero [48].

\subsubsection{Quantification of the density of vessels}

The number of vessels was quantified at days 7 and 14 postoperative in the CD31 stained samples. Five non-consecutive tissue sections per time point were randomly selected for each group. Quantification and measurement were performed in high-power fields, and only vessels with a diameter $<50 \mu \mathrm{m}$ were considered [49]. The results of the number of vessels per field are expressed as density of vessels.

\subsubsection{Epidermal thickness measurement}

The thickness of epidermal fraction of the neoskin was evaluated at 7, 14 and 21 days post-operative in $\mathrm{H} \& \mathrm{E}$ routinely stained histological sections. Four sections per group and time point were analysed by randomly selecting five high-power fields and performing five measurements of the epidermal thickness per field.

\subsection{Statistical analysis}

Four animals $(n=4)$ were used in each group for each time point. Standard deviation is reported as a measure of sample deviation. Statistical analysis of wound closure was performed using two-way analysis of variance (ANOVA) with Bonferroni post-tests. The density of vessels data were analysed by one-way ANOVA with
Tukey's post-tests, while the epidermal thickness data were analysed by Kruskal-Wallis test, with Dunn's post-test (GraphPad Prism 4.02). Significance levels between groups, determined using post-tests, were set for $P<0.05$.

\section{Results}

\subsection{In vitro phenotypic characterization of single CS and 3-D CS-based constructs}

A set of single hDFb CS, as well as homotypic and heterotypic $\mathrm{CS}$, formed by hDFb with either hDMEC or hKC cultured on top, were successfully achieved after 5 days of culture in thermoresponsive culture dishes (Fig. $2 \mathrm{iA}$ ) and harvested by temperature decrease (Fig. 2iB), without the need for enzymatic treatment. The different types of cells composing each of the homotypic and heterotypic CS and respective arrangement within the co-cultures was confirmed by the expression of the specific markers by immunohistochemistry prior (Fig. 2iA) and after (Fig. 2iB) detachment. hDMEC were identified by their capacity to uptake Dil-AC-LDL and by the expression of $\mathrm{vWF} / \mathrm{CD} 31$. After 2 days of culture on top of a confluent layer of hDFb, hDMEC exhibiting the characteristic cobblestone morphology were organized in colonies (Fig. 2iA). The hKC expressing K14 displayed a random distribution on top of cultured hDFb, distinguished as the cells only stained with DAPI in Fig. 2iA and expressing FSP in Fig. 2iB. The co-cultured CS cross sections (Fig. 2iB) allowed confirmation of the hKC and the hDMEC on top of the hDFb embedded in their own ECM.

The adhesive nature of single CS, conferred by the ECM deposited by the hDFb, allowed their assembly into different types of 3-D constructs. After stacking, the CS-based constructs showed great stability, as revealed by the contiguous 2-CS layered structure (Fig. 2ii). The cross sections of the 3-D constructs generated confirmed their desired design and the localization of the different types of cells within the structure. The presence of hDMEC on the upper part and between the two hDFb layers of the 3-D construct composed of two single CS of co-cultured hDFb and hDMEC (hDFb + hDMEC-CS) was confirmed by the expression of CD31 (Fig. 2ii). Similarly, the construct composed of one hDFb CS and a second one of hDFb co-cultured with hKC (hDFb + hKC-CS) shows the epidermal cells expressing K14 on the top of construct. Lastly, the expected arrangement of the 3-D construct created by the combination of a CS of co-cultured hDFb and hDMEC, and a second $\mathrm{CS}$ of hDFb co-cultured with hKC (hDFb+hDMEC + hKC-CS), resembling native skin organization with hDMEC embedded in the hDFb matrix and hKC localized on the construct top layer, is demonstrated (Fig. 2ii).

\subsection{Effect of composition of CS-based skin constructs on wound closure}

The proposed CS-based constructs showed a different influence over wound closure at different time points, as confirmed by macroscopic analysis (Fig. 3i) and closure measurements (Fig. 3ii). While for days 3 and 7 postoperative the percentage of wound closure did not vary between the test conditions, at day 14 the 3 -D constructs composed of the two CS of hDFb and hDMEC led to the highest percentage of wound closure $(82 \% \pm 8)$, significantly different $(P<0.05)$ from the control $(65 \% \pm 3)$. From day 14 onwards, wounds were progressively closing, reaching total closure at day 21 postoperative for all the conditions. At the final time point, the CS-based constructs combining the hDFb with hKC $(95 \% \pm 1)$ and the hDFb plus hDMEC and hKC $(97 \% \pm 3)$, aided a significantly $(P<0.05)$ higher wound closure percentage than the control $(78 \% \pm 14)$ (Fig. 3ii). 
(i)

$$
\text { Single CS }
$$

\section{A}

CS Prior Detachment

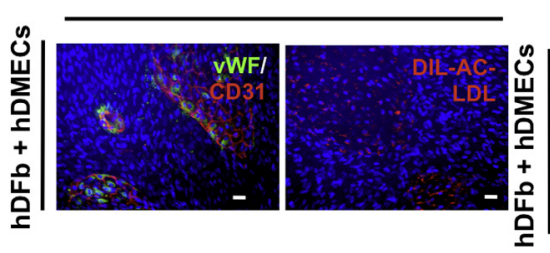

B Organization within ECM crosssection

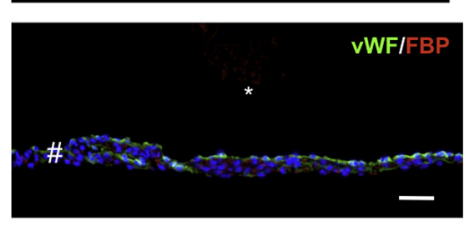

(ii)

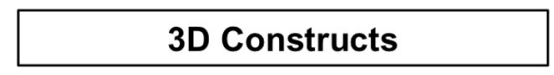

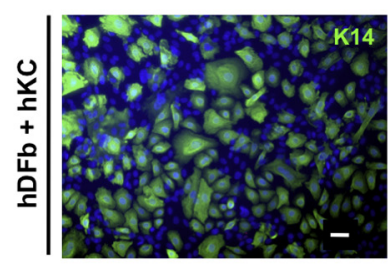
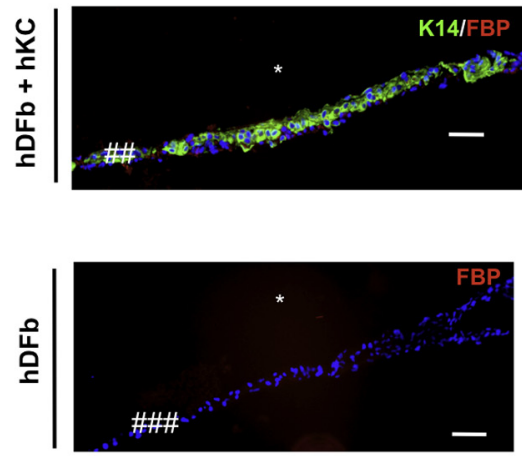
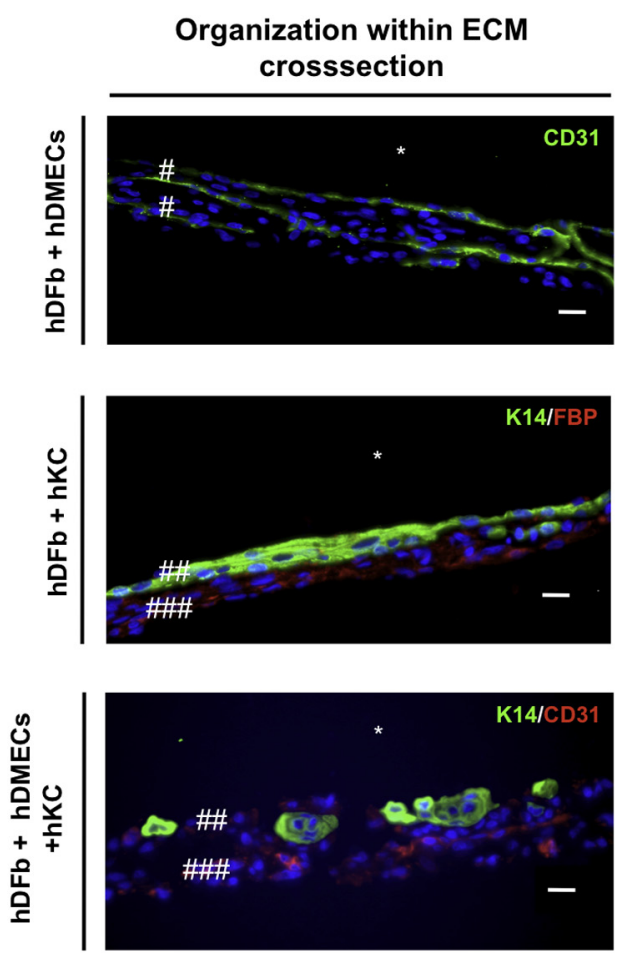

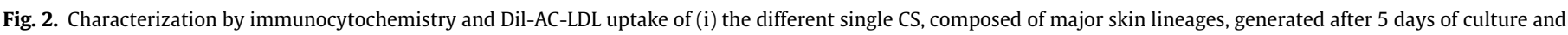

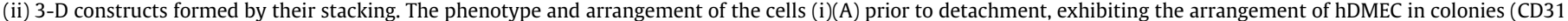

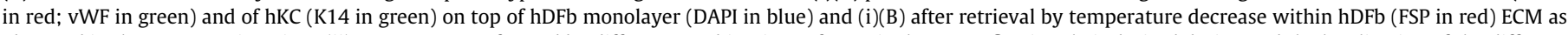

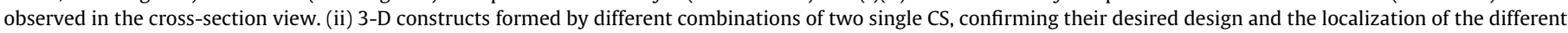

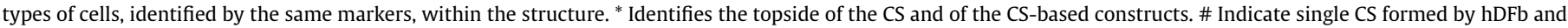
hDMEC, \#\# single CS with co-cultured hKC and hDFb, and \#\#\# single CS formed only by hDFb. Scale bars represent $50 \mu \mathrm{m}$.

Detailed wound healing analysis, as visualized after H\&E staining (Fig. 3iii), revealed the beginning of the formation of a neoepidermis, noticeable underneath the eschar, at day 7 for all the experimental groups (Fig. 3iiiF, J, N). In contrast, in the control condition, a great extent of granulation tissue was visible, with an eschar starting to form. A neoepidermis, similar to that observed at day 7 for the experimental groups, was only noted at day 14 for the control (Fig. 3iiiB, C). The granulation tissue acted as a template for a vascularized neodermis formation from day 7 onwards, appearing to be more organized for the experimental groups from day 14 postoperative (Fig. 3iiiG, K, O). Nonetheless, a complete reepithelialization of the wounds was observed for all the conditions tested at day 21 (Fig. 3iiiD, H, L, P). In order to analyse the re-epithelialization of the wounds in more detail, the thickness of the neoepidermis at 7 (Fig. 4iA), 14 (Fig. 4iB) and 21 days (Fig. $4 \mathrm{iC}$ ) was measured. At 14 days postoperative, the CS-based constructs containing hDFb plus hDMEC, in either the presence or absence of hKC, led to a significantly thicker $(P<0.05)$ neoepidermis than both the control and the CS-based construct containing hDFb and hKC $(P<0.01$ and $P<0.001)$. At day 21 , all the experimental groups exhibited similar neoepidermis thickness, significantly thicker $(P<0.05)$ than the control, and closer to the thickness of healthy mouse epidermis (Fig. S1).

\subsection{Human cells from CS-based constructs engraft into host neotissue and enhance vascularization}

The transplanted CS-based constructs immediately adhered to the wound bed. In Fig. $5 \mathrm{i}$, it is possible to observe the transplanted
CS on top of the wound, creating a continuous layer that overlaps the wound margins, guaranteeing uniform integration into the wound bed. At day 3, the human cells were distributed within the wound site, with special prevalence at the wound margins (Fig. 5iiA, B), independently of the experimental condition. Although the design of the transplanted CS could not be clearly identified, at day 3, clusters of human cells corresponding to the skin cells that integrated the implanted CS-based constructs were localized. The presence of hDMEC (Fig. 5 iiiA, D) and hKC (Fig. 5iiiB, C), expressing human CD31 and K14, respectively, was confirmed in the corresponding constructs. The predominance of human cells at wound margins was even more evident at day 7; human cells were mostly, but not exclusively, found in wound borders (Fig. 5iiD, E). In contrast to what was observed at day 3, groups of human cells were only found on the top centre of the wound for the hDFbs + hKC-CS group, in what appears to be the newly formed epidermis. At day 14, isolated human cells were still visible within the neoformed tissue (Fig. 5iiG-I), although in lower numbers. At day 21, almost no cells were found within the tissue (data not shown).

The impact of the transplanted hDMEC over the progression of wound healing and, in particular, over neovascularization, was analysed by determining the density of the vessels within the wounded area at days 7 and 14 (Fig. 6). An effect dependent on the composition of the constructs over the density of vessels was observed at day 7, but not at day 14 post implantation. At the earlier time point, the density of vessels was significantly higher $(P<0.05)$ for the constructs containing CS of co-cultured hDFb and hDMEC condition than for the CS-based constructs containing 

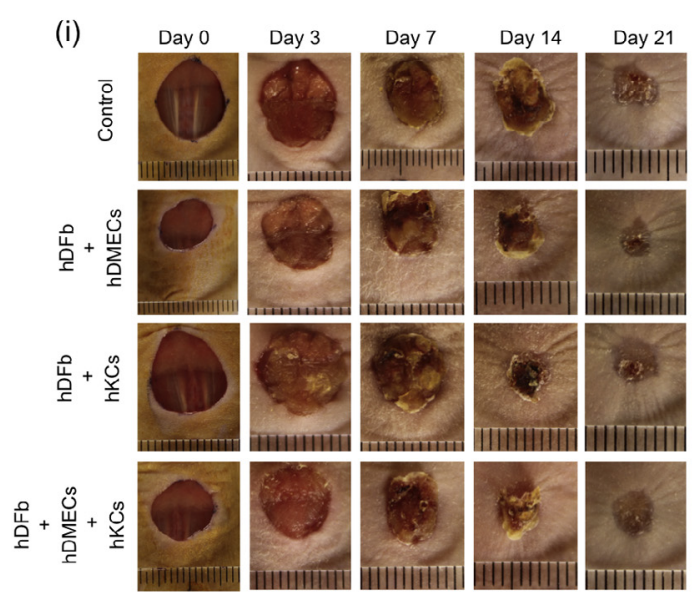

(ii)
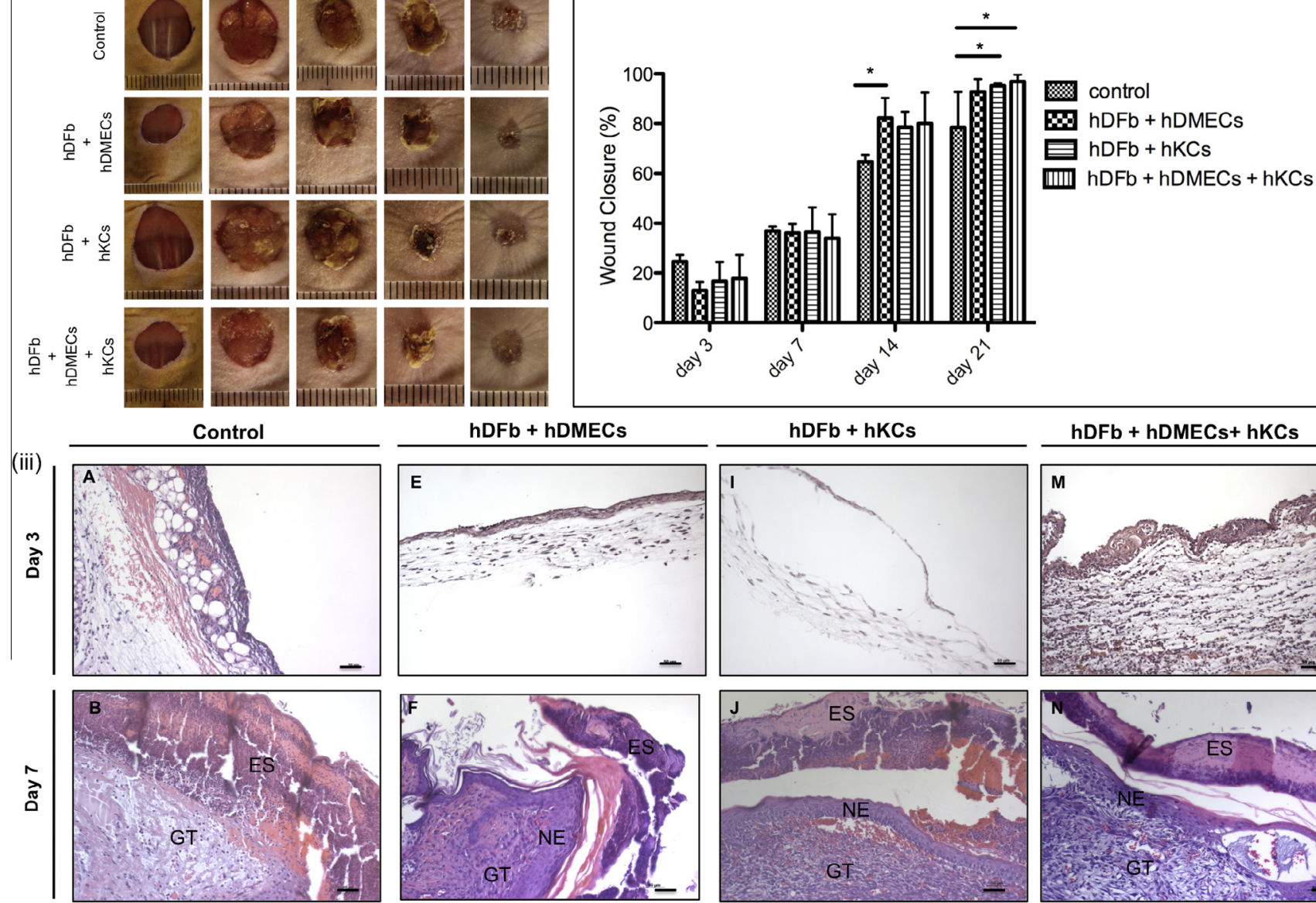

hDFb + hDMECs+ hKCs
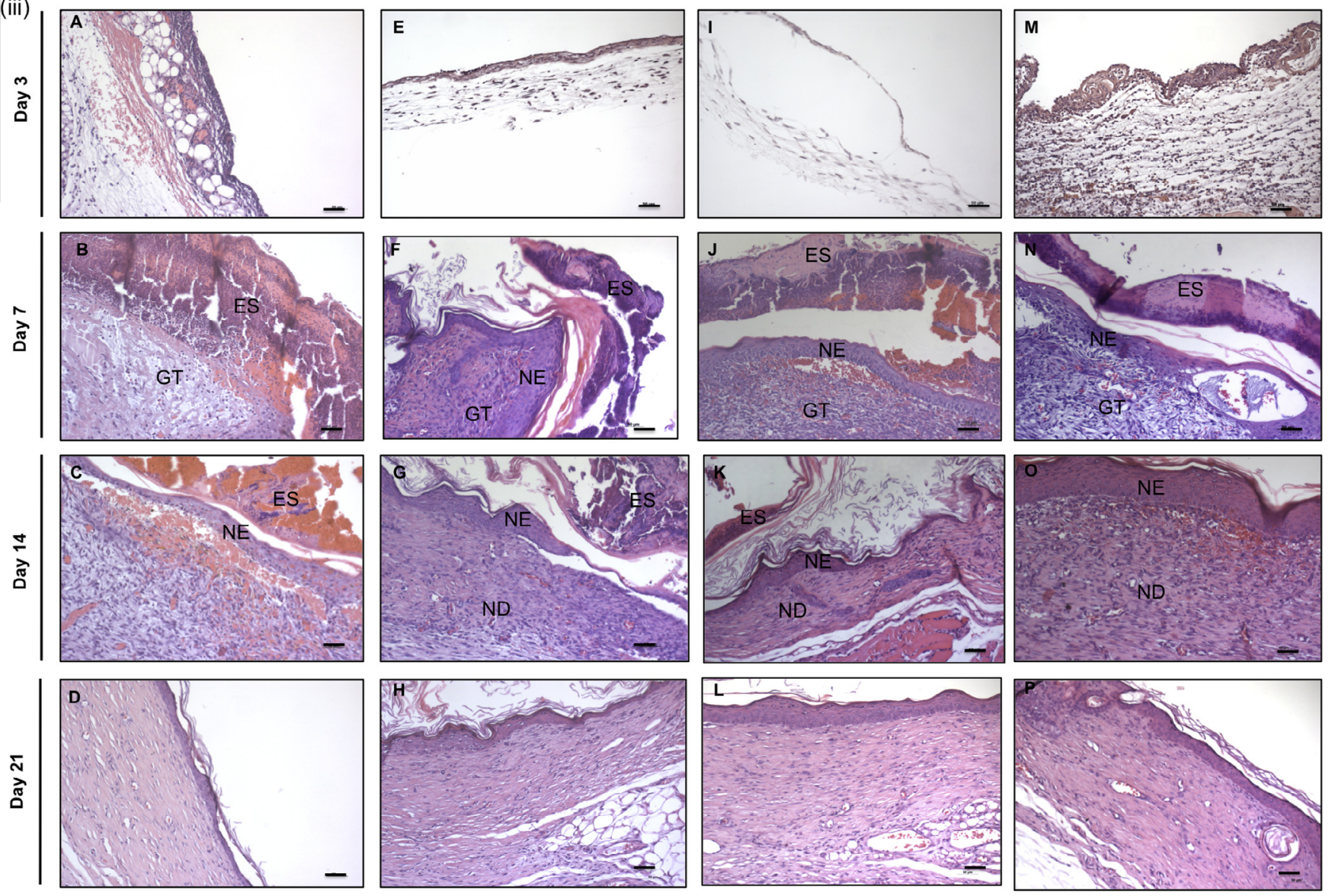

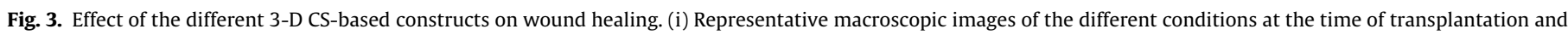

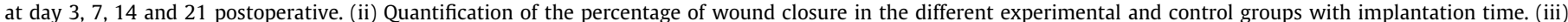

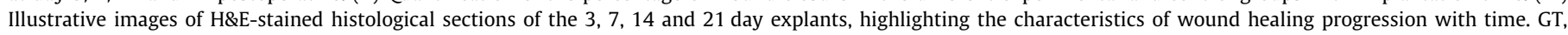
granulation tissue; ES, eschar; NE, neoepidermis; ND, neodermis. Scale-bar represents $50 \mu \mathrm{m}$.

hDFb and hKC $(P<0.001)$ and the control $(P<0.01)$ groups (Fig. 6iA). Moreover, a significantly higher density of vessels was also detected for the group of CS-based constructs combining hDFb, hDMEC and hKC $(P<0.05)$ in relation to the CS-based constructs combining hDFb and hKC, but not in comparison with the control $(P>0.05)$. At day 14 , no significant differences in the density of vessels were detected among the different groups (Fig. 6iB). The contribution of the transplanted hDMEC for the formation of new blood vessels was reinforced by the identification within the neovasculature of human CD31 positive cells at day 14 (Fig. 6ii).

\section{Discussion}

Effective wound healing involves a well-orchestrated multidirectional interaction between several cellular players and environmental cues to progressively restore skin barrier function through matrix deposition/remodelling, vascularization and re-epithelialization [50,51]. The present study explored the use of CS technology to create CS formed by relevant skin lineages, which were then assembled as different 3-D CS-based constructs. Moreover, it took advantage of the possibility of accurately defining the composition 


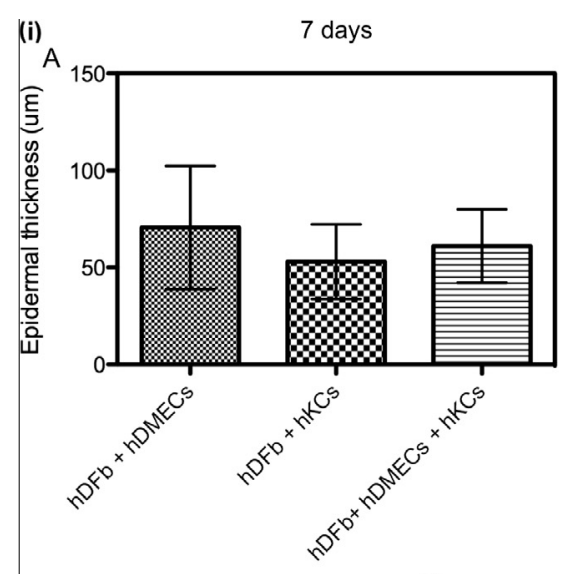

(ii)
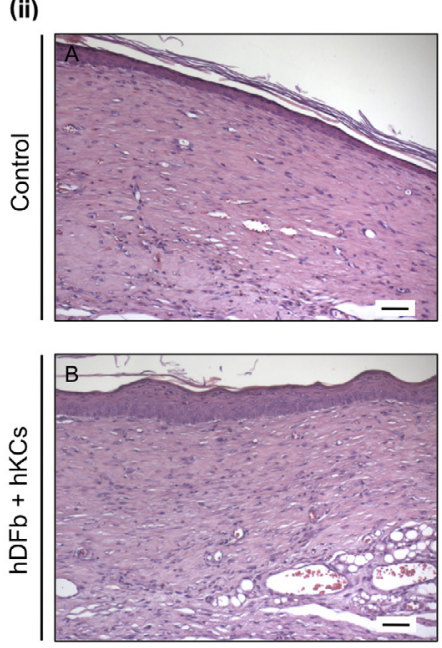

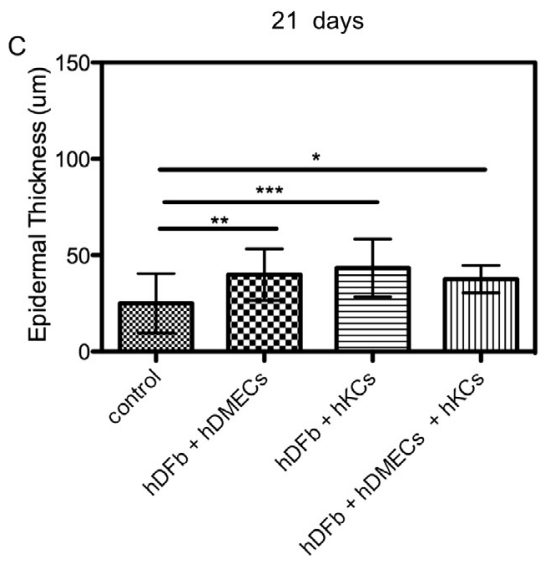

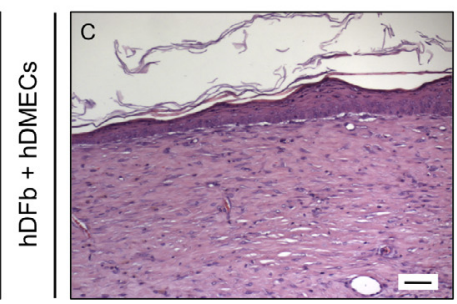

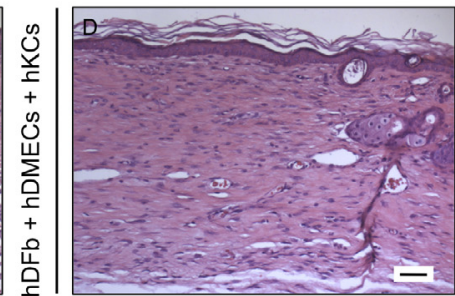

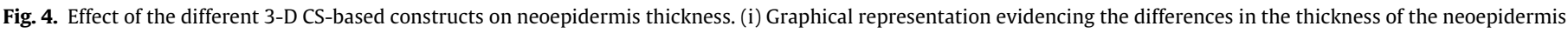

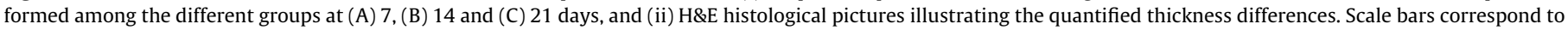
$50 \mu \mathrm{m} .{ }^{*} \mathrm{P}<0.05 ;{ }^{* *} \mathrm{P}<0.01 ;{ }^{* * *} \mathrm{P}<0.001$.

and organization of the CS-based constructs to specifically target re-vascularization and re-epithelialization during the progression of wound healing.

One of the requirements for CS retrieval from thermo-responsive surfaces is the deposition of a robust ECM that embeds cells within their own matrix, forming a sheet [52]. The same skin sample allowed the three main skin lineages to be obtained to produce the CS. However, both hKC and hDMEC are unable to form a thick ECM in vitro and are also particularly sensitive in terms of culture conditions [53,54]. Moreover, the selection of pure cultures of hDMEC involves extensive cell manipulation, discouraged many times owing to fibroblast contamination [43]. Similarly, the prolonged culture of KC leads to terminal differentiation [42]. In the sequence of these constraints, and with the prospect of achieving suitable constructs in a short time-frame, the present authors propose a strategy in which hDFb, in all the constructs, produce an ECM-rich template to co-culture hKC and hDMEC. Either keratinocytes or endothelial cells were randomly distributed on the hDFb and embedded in the ECM. Thus, on stacking and due to the natural adhesiveness of the CS, cells deeply interact, as demonstrated by the apparent fused layers observed in the cross section of the heterotypic constructs. The proposed approach not only guaranteed the production of a robust CS and its retrieval from the thermoresponsive dishes, but also the direct interaction of hKC and hDMEC with the hDFb, the most important modulator of wound healing signalling in vivo [51]. In fact, interactions between hKC and hDFb are crucial for skin regeneration; hKC stimulate hDFb to synthesize growth factors such as IL-1 alpha/beta, which in turn affect hKC in a double paracrine manner [51]. Additionally, the role of hDFb in neovascularization, namely in their differentiated form as myofibroblasts, is widely acknowledged. Myofibroblasts are known to deposit large amounts of ECM, the framework required for new vessel formation by endothelial cells [55]. In addition, through the release of different cytokines, such as bFGF and TGF $\beta 1$, as well as matrix metalloproteinases, myofibroblasts induce the proliferation and migration of endothelial cells and, consequently, angiogenesis/neovascularization [56].

Significant wound contraction is often associated with the rodent model used here [57]. Despite the administration of a steroid to attenuate wound contraction [58] and to delay the healing process [47], considerable contraction was still observed. However, the present authors consider that, as similar degree of contraction was observed for all the conditions, direct comparison of the results between the experimental groups was not compromised. CS-based constructs were designed considering not only the described interplay between the cells involved, but particularly the specific role of $\mathrm{KC}$ and endothelial cells in re-epithelialization and neovascularization, respectively. Stable CS-based constructs were obtained, and their compositions had different impacts on the healing progression of mouse full-thickness excisional wounds. Although a faster wound closure was observed with the construct combining two CS of hDFb and hDMEC, the percentage of wound closure at the end time point of the assay was significantly higher in comparison with the control, for the conditions incorporating 
(iii)

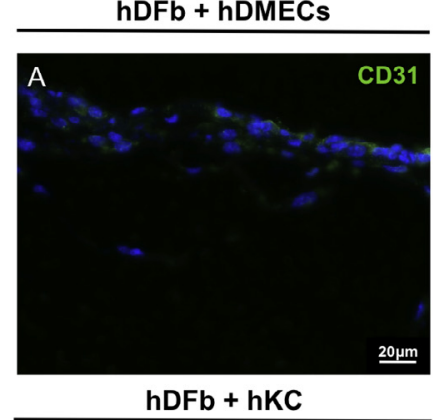

(i)
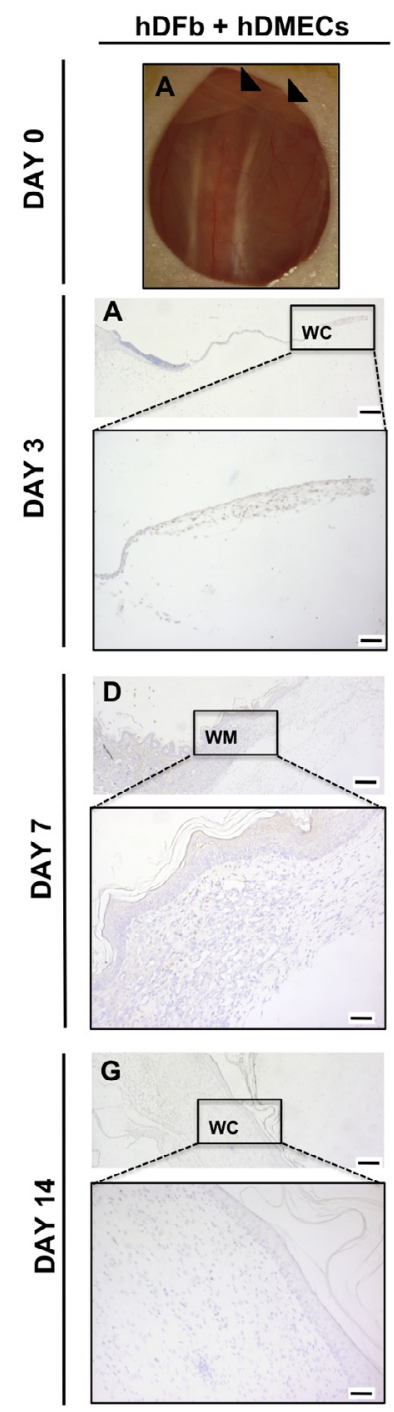

hDFb + hKC

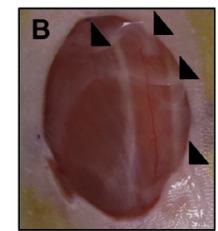

B
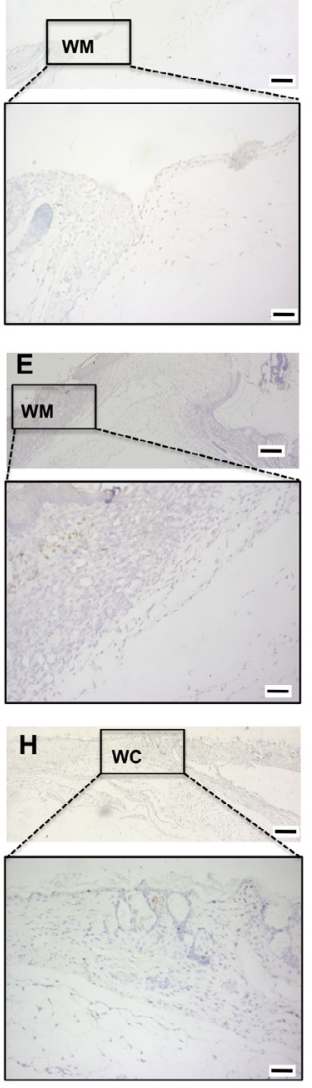

hDFb + hDMECs + hKC
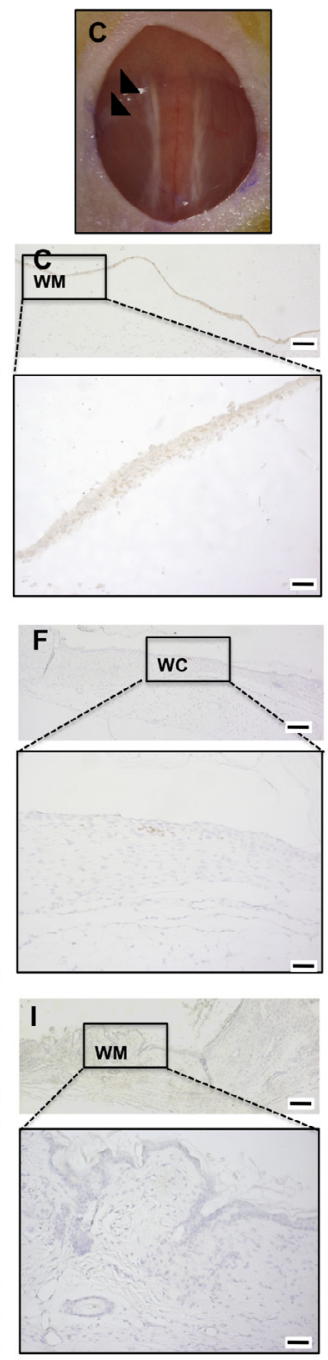

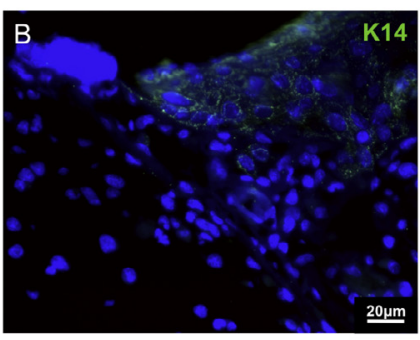

$\mathrm{hDFb}+\mathrm{hDMECs}+\mathrm{hKC}$
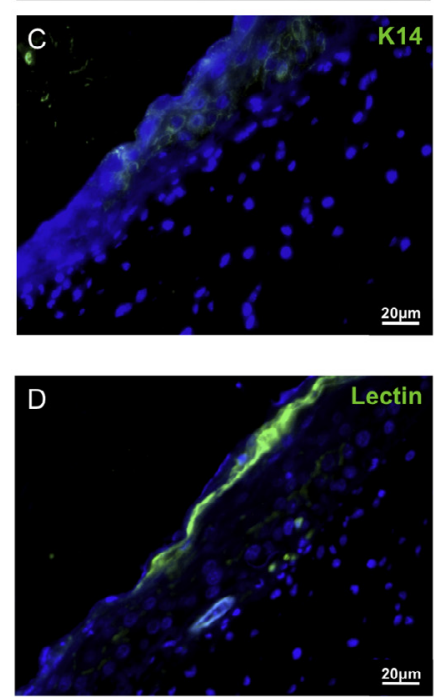

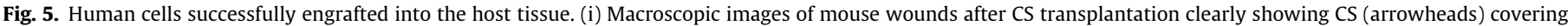

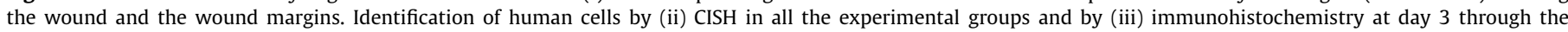

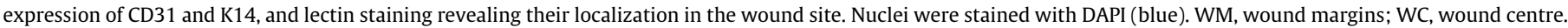

hKC. In a wound healing scenario, the thickness of the neoepidermis correlates with the re-epithelialization process, which implies increased keratinocyte proliferation and consequent epidermis thickening and keratinization, gradually establishing homeostasis [59]. No significant differences between the different experimental conditions, in terms of epidermis thickness, were observed at the end time point, suggesting re-establishment of the homeostatic equilibrium. However, the progression of re-epithelialization varied with the composition of the constructs. In agreement with the wound closure rate, faster re-epithelialization, traduced in a thicker epidermis, was observed when hKC integrated the constructs formed with a second CS of hDFb co-cultured with hDMEC. Additionally, a similar wound closure percentage and epidermal thickness were observed for the groups containing hDMEC, which suggests a contribution of these cells to re-epithelialization. These observations meet previous findings [60] that underlined the close interaction between the different cell types forming the proposed constructs. The effect of the presence of endothelial cells over KC migration, consequently enhancing re-epithelialization, traduced in thicker neoepidermis, was demonstrated. Moreover, both hDFb and hDMEC are able to express factors, namely keratinocyte growth factor and hepatocyte growth factor, that are known to stimulate $\mathrm{KC}$ proliferation and migration. Unexpectedly, the constructs only combining hDFb and hKC did not impact early re-epithelialization, which might be related to the number of hKC used [5].

The rationale for the design of constructs containing hDMEC, only in-between the hDFb and in-between and on one side of the construct for the direct interaction with the wound bed after transplantation, was to promote angiogenesis and faster neovascularization. Endothelial cells are known to constitutively express vascular endothelial factors and to be able to respond to the wound environment, enhancing neovascularization [61-63]. Furthermore, as for other TE approaches, the incorporation of endothelial cells within the 3-D CS-based constructs has been proved successful in this respect. Both endothelial homotypic [64] and heterotypic 
(i)

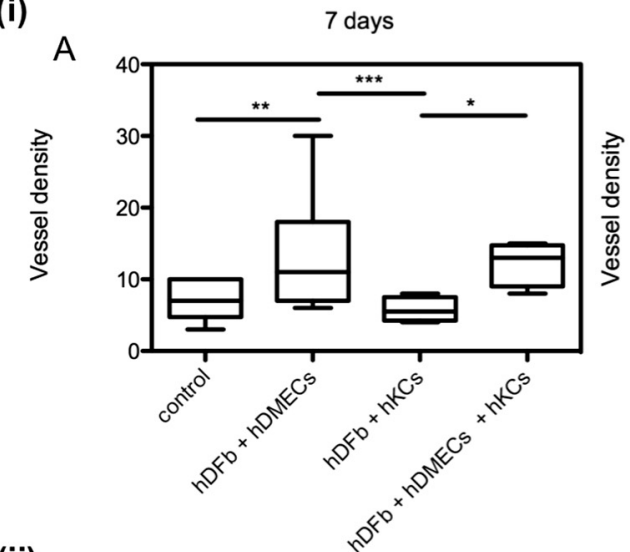

(ii)

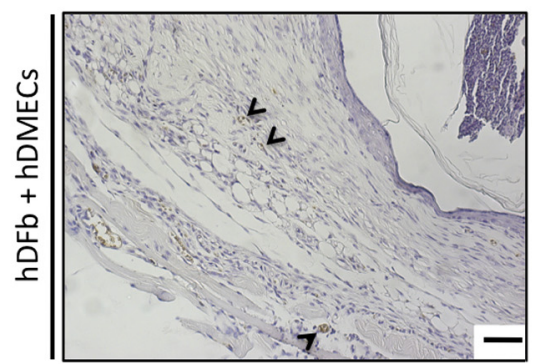

B
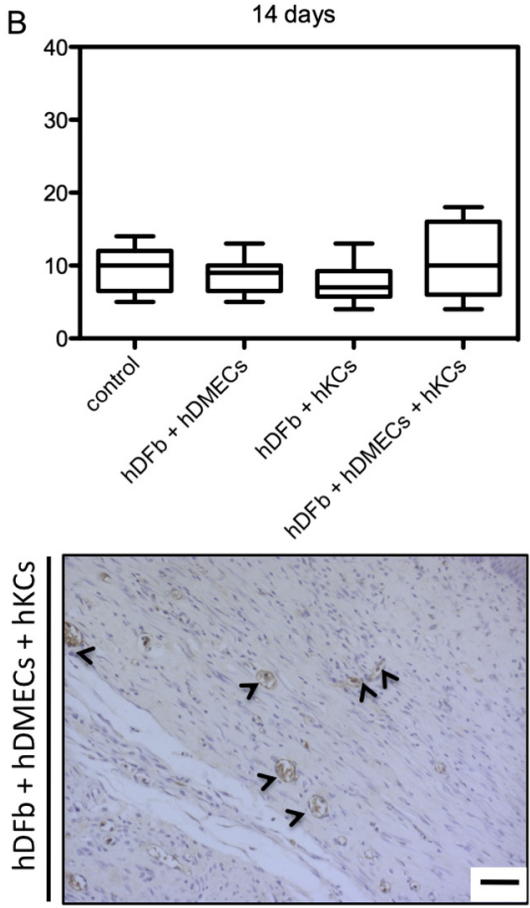

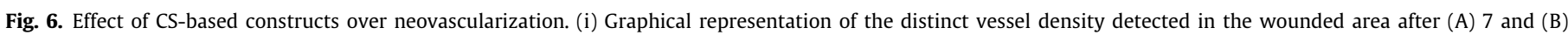

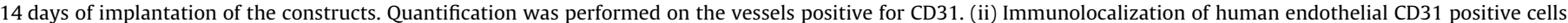
(arrow heads) within the neovasculature formed. ${ }^{*} P<0.05$; ${ }^{* *} P<0.01 ;{ }^{* * *} P<0.001$.

CS obtained after co-culturing endothelial cells with fibroblasts on thermo-responsive surfaces $[39,64-66]$ have been used to create a variety of pre-vascularized structures and to promote in vivo neovascularization. The hDMEC cultured on top of the confluent hDFb organized in colonies, which have been suggested to act as nucleation sites for the later formation of tube-like structures in vitro [67]. Moreover, there are indications that hDFb and endothelial cell interaction in vitro promote the formation and maintenance of capillary-like structures $[21,64,68]$, as hDFb are thought to recreate physiological conditions $[64,69]$. As one of the present authors' major aims was to propose a strategy with a minimum in vitro culture period, thus accelerating the constructs' readiness for transplantation, they were not able to demonstrate the in vitro formation of capillary-like structures, in contrast to other studies that demonstrated this event in prolonged in vitro culture $[21,70]$. However, a significant increase in the number of vessels was observed within the wounded area at early time points for both CS-based constructs containing hDMEC in their composition. Two main mechanisms may be contributing to this effect; the early trophic release of angiogenic factors by the transplanted cells [60], and the later integration of human endothelial cells in the neovasculature. Regarding the release of angiogenic factors, it would be expected that an increase in the number of transplanted hDMEC would enhance its release and, consequently, its effect. While the ratios between $\mathrm{hDFb}$ and hDMEC were maintained among the CS, the construct composed of two stacked CS of hDFb co-cultured with hDMEC contained a higher number of hDMEC than the construct composed of one CS of hDFb co-cultured with hDMEC and a second CS of hDFb co-cultured with hKC. This higher total number of endothelial cells did not result in a significant difference in vessel density between the two conditions, which is in agreement with studies by Asakawa et al. [64]. Moreover, the dynamic wound healing process with consecutive steps, formation of granulation tissue, re-epithelialization and neodermis matrix deposition, leads to extensive remodelling at the wound site. Thus, as a result of matrix remodelling and displacement of the transplanted cells over time, the trophic release of angiogenic factors effect is overtaken by host mediated signalling at later time points. Thus, the contribution of the transplanted hDMEC evolves from an indirect role within the healing environment to a direct function, as part of the neovasculature as healing progresses.

The common outcome and limitation of in vivo xenotransplantation, which is a diminishing number of transplanted human cells with time, was also observed with the present approach. Their dissociated distribution, and not in a CS organization, was observed within the neotissue formed. However, and in accordance with others, the cells transplanted as CS impacted wound healing at early time points and, therefore, the way it progressed. This has been proved to occur as a result of the trophic effect of the transplanted cells through paracrine interactions with resident cells $[29,51,60,71]$. Overall, the present authors were able to confirm the hypothesis that the combination of homotypic and heterotypic CS, composed of the skin relevant cell lineages, into 3-D CS-based structures provides the necessary cell machinery for wound healing, specifically influencing re-vascularization and re-epithelialization. These findings support the theory of cell action through paracrine signalling and eliminates the need for long-term survival of the transplanted cells.

\section{Conclusion}

The present authors demonstrated that different homotypic and heterotypic CS, formed by skin cells, could be generated from thermo-responsive surfaces and assembled into distinctly designed 3-D constructs in a short time-frame, which is important when envisioning rapid application in a clinical setting. Moreover, the findings suggest that the composition of the 3-D constructs affected the healing of full-thickness excisional wounds, leading to distinct outcomes. Although absent at the end of the study, the transplanted human cells observed in the host wound bed and within the neotissue 
formed contributed to the progress of re-epithelialization and neovascularization of the wounded tissue. While re-epithelialization was affected by the transplanted hKC and hDMEC, neovascularization was directly influenced by the presence of hDMEC within the constructs. Synergistic interaction of the different cells transplanted within the ECM produced by the hDFb towards fast wound reepithelialization is suggested. Moreover, the contribution of hDMEC to improving neotissue vascularization was also confirmed. Altogether, these results warrant further studies using the proposed strategy in the pursuit of improved skin regeneration.

\section{Acknowledgements}

The authors thank Hospital da Prelada (Porto), in particular Dr. Paulo Costa for lipoaspirate collection, and Skingineering (PTDC/ SAU-OSM/099422/2008) for to financial support; a Portuguese Foundation for Science and Technology (FCT) funded project. The research leading to these results has also received funding from the European Union's Seventh Framework Programme (FP7/20072013) under grant agreement REGPOT-CT2012-316331-POLARIS.

There are no competing financial interests.

\section{Appendix A. Figures with essential colour discrimination}

All figures in this article, Figs. 1-6, are difficult to interpret in black and white. The full colour images can be found in the on-line version, at http://dx.doi.org/10.1016/j.actbio.2014.03.006

\section{Appendix B. Supplementary data}

Supplementary data associated with this article can be found, in the online version, at http://dx.doi.org/10.1016/j.actbio.2014.03. 006.

\section{References}

[1] Rheinwald JG, Green H. Serial cultivation of strains of human epidermal keratinocytes: the formation of keratinizing colonies from single cells. Cell 1975;6(3):331-43.

[2] O'Connor NE et al. Grafting burns with cultured epithelium prepared from autologous epidermal cells. Lancet 1981;8211:75-8.

[3] Faure M et al. Growth and differentiation of human epidermal cultures used as auto- and allografts in humans. Br J Dermatol 1987;116(2):161-70.

[4] Madden MR et al. Grafting of cultured allogeneic epidermis on second- and third-degree burn wounds on 26 patients. J Trauma 1986;26(11):955-62.

[5] Phillips TJ et al. Cultured epidermal autografts and allografts: a study of differentiation and allograft survival. J Am Acad Dermatol 1990;23(2 Pt 1):189-98

[6] Auxenfans $C$ et al. Use of allogenic epidermal sheets for difficult wound healing: selection and testing of relevant growth factors. Biomed Mater Eng 2006;16(4 Suppl.):S73-83.

[7] Terskikh VV, Vasiliev AV. Cultivation and transplantation of epidermal keratinocytes. Int Rev Cytol 1999;188:41-72.

[8] Fratianne R et al. Keratinocyte allografts accelerate healing of split-thickness donor sites: applications for improved treatment of burns. J Burn Care Rehabil 1993;14(2 Pt 1):148-54.

[9] Beele $\mathrm{H}$ et al. Repeated cultured epidermal allografts in the treatment of chronic leg ulcers of various origins. Dermatologica 1991;183(1):31-5.

[10] Pye RJ. Cultured keratinocytes as biological wound dressings. Eye (Lond) 1988;2(Pt 2):172-8.

[11] Tamariz-Dominguez E, Castro-Munozledo F, Kuri-Harcuch W. Growth factors and extracellular matrix proteins during wound healing promoted with frozen cultured sheets of human epidermal keratinocytes. Cell Tissue Res 2002;307(1):79-89.

[12] Shirakata Y et al. So-called biological dressing effects of cultured epidermal sheets are mediated by the production of EGF family, TGF-beta and VEGF. J Dermatol Sci 2003;32(3):209-15.

[13] Desai $\mathrm{MH}$ et al. Lack of long-term durability of cultured keratinocyte burnwound coverage: a case report. J Burn Care Rehabil 1991;12(6):540-5.

[14] Tausche AK et al. An autologous epidermal equivalent tissue-engineered from follicular outer root sheath keratinocytes is as effective as split-thickness skin autograft in recalcitrant vascular leg ulcers. Wound Repair Regen 2003;11(4):248-52.
[15] Wood FM, Kolybaba ML, Allen P. The use of cultured epithelial autograft in the treatment of major burn wounds: eleven years of clinical experience. Burns 2006;32(5):538-44.

[16] Atiyeh BS, Gunn SW, Hayek SN. State of the art in burn treatment. World J Surg 2005;29(2):131-48.

[17] Cuono C, Langdon R, McGuire J. Use of cultured epidermal autografts and dermal allografts as skin replacement after burn injury. Lancet 1986;1(8490): $1123-4$.

[18] Kangesu T et al. Kerato-dermal grafts: the importance of dermis for the in vivo growth of cultured keratinocytes. Br J Plast Surg 1993;46(5):401-9.

[19] Krejci-Papa NC, Hoang A, Hansbrough JF. Fibroblast sheets enable epithelialization of sounds that do not support keratinocyte migration. Tissue Eng 1999;5(6):555-62.

[20] Pouliot R et al. Reconstructed human skin produced in vitro and grafted on athymic mice. Transplantation 2002;73(11):1751-7.

[21] Liu Y et al. In vitro construction of scaffold-free bilayered tissue-engineered skin containing capillary networks. Biomed Res Int 2013;2013:561410.

[22] Rochon $\mathrm{MH}$ et al. Normal human epithelial cells regulate the size and morphology of tissue-engineered capillaries. Tissue Eng Part A 2010;16(5): 1457-68.

[23] Nishida K et al. Corneal reconstruction with tissue-engineered cell sheets composed of autologous oral mucosal epithelium. N Engl J Med 2004:1187-96.

[24] Shimizu T et al. Fabrication of pulsatile cardiac tissue grafts using a novel 3dimensional cell sheet manipulation technique and temperature-responsive cell culture surfaces. Am Heart Assoc 2002;90:e40-8.

[25] Hasegawa $M$ et al. Human periodontal ligament cell sheets can regenerate periodontal ligament tissue in an athymic rat model. Tissue Eng 2005;11(3-4): 469-78.

[26] Shiroyanagi Y et al. Transplantable urothelial cell sheets harvested noninvasively from temperature-responsive culture surfaces by reducing temperature. Tissue Eng 2003;9(5):1005-12.

[27] Yamato $\mathrm{M}$ et al. Thermo-responsive culture dishes allow the intact harvest of multilayered keratinocyte sheets without dispase by reducing temperature. Tissue Eng 2001;7(4):473-80.

[28] McLaughlin MM, Marra KG. The use of adipose-derived stem cells as sheets for wound healing. Organogenesis 2013;9(2):79-81.

[29] Cerqueira MT et al. Human adipose stem cells cell sheet constructs impact epidermal morphogenesis in full-thickness excisional wounds. Biomacromolecules 2013;14:3997-4008.

[30] Yang J et al. Reconstruction of functional tissues with cell sheet engineering. Biomaterials 2007;28(34):5033-43.

[31] Haraguchi $Y$ et al. Fabrication of functional three-dimensional tissues by stacking cell sheets in vitro. Nat Protoc 2012;7(5):850-8.

[32] Sekiya S et al. Bioengineered cardiac cell sheet grafts have intrinsic angiogenic potential. Biochem Biophys Res Commun 2006;341(2):573-82.

[33] Koga Y et al. Recovery course of full-thickness skin defects with exposed bone: an evaluation by a quantitative examination of new blood vessels. J Surg Res 2007;137(1):30-7.

[34] Ohashi $\mathrm{K}$ et al. Engineering functional two- and three-dimensional liver systems in vivo using hepatic tissue sheets. Nat Med 2007;13(7):880-5.

[35] Masuda S et al. Cell sheet engineering for heart tissue repair. Adv Drug Deliv Rev 2008;60(2):277-85.

[36] Iwata $\mathrm{T}$ et al. Periodontal regeneration with multi-layered periodontal ligament-derived cell sheets in a canine model. Biomaterials 2009;30(14): 2716-23.

[37] Sekiya $N$ et al. Layered implantation of myoblast sheets attenuates adverse cardiac remodeling of the infarcted heart. J Thorac Cardiovasc Surg 2009;138(4):985-93.

[38] Asakawa $\mathrm{N}$ et al. Pre-vascularization of in vitro three-dimensional tissues created by cell sheet engineering. Biomaterials 2011;31(14):3903-9.

[39] Mendes LF et al. Perivascular-like cells contribute to the stability of the vascular network of osteogenic tissue formed from cell sheet-based constructs. PLoS One 2012;7(7):e41051.

[40] Sekine $\mathrm{H}$ et al. Cardiac cell sheet transplantation improves damaged heart function via superior cell survival in comparison with dissociated cell injection. Tissue Eng Part A 2011;17(23-24):2973-80.

[41] Lin YC et al. Evaluation of a multi-layer adipose-derived stem cell sheet in a full-thickness wound healing model. Acta Biomater 2012;9(2):5243-50.

[42] Barrandon Y, Green H. Three clonal types of keratinocyte with different capacities for multiplication. Proc Natl Acad Sci U S A 1987;84(8):2302-6.

[43] Richard L, Velasco P, Detmar M. A simple immunomagnetic protocol for the selective isolation and long-term culture of human dermal microvascular endothelial cells. Exp Cell Res 1998;240(1):1-6.

[44] Moll I et al. Characterization of epidermal wound healing in a human skin organ culture model: acceleration by transplanted keratinocytes. J Invest Dermatol 1998;111(2):251-8.

[45] MacNeil S. What role does the extracellular matrix serve in skin grafting and wound healing? Burns 1994;20(Suppl. 1):S67-70.

[46] Supp DM, Wilson-Landy K, Boyce ST. Human dermal microvascular endothelial cells form vascular analogs in cultured skin substitutes after grafting to athymic mice. FASEB J 2002;16(8):797-804.

[47] Wicke C et al. Effects of steroids and retinoids on wound healing. Arch Surg 2000;135(11):1265-70.

[48] Ananta M, Brown RA, Mudera V. A rapid fabricated living dermal equivalent for skin tissue engineering: an in vivo evaluation in an acute wound model. Tissue Eng Part A 2012;18(3-4):353-61. 
[49] Liu S et al. Synergistic angiogenesis promoting effects of extracellular matrix scaffolds and adipose-derived stem cells during wound repair. Tissue Eng Part A $2011 ; 17(5-6): 725-39$

[50] Hughes CC. Endothelial-stromal interactions in angiogenesis. Curr Opin Hematol 2008;15(3):204-9.

[51] Werner S, Krieg T, Smola H. Keratinocyte-fibroblast interactions in wound healing. J Invest Dermatol 2007;127(5):998-1008.

[52] Okano T et al. A novel recovery system for cultured cells using plasma-treated polystyrene dishes grafted with poly(N-isopropylacrylamide). J Biomed Mater Res 1993;27(10):1243-51.

[53] Clark RA et al. Human keratinocytes that have not terminally differentiated synthesize laminin and fibronectin but deposit only fibronectin in the pericellular matrix. J Cell Biochem 1985;28(2):127-41.

[54] Kramer RH et al. Basal lamina formation by cultured microvascular endothelial cells. J Cell Biol 1984;99(2):692-8.

[55] Singer AJ, Clark RA. Cutaneous wound healing. N Engl J Med 1999;341(10): $738-46$.

[56] Oberringer $\mathrm{M}$ et al. A new in vitro wound model based on the co-culture of human dermal microvascular endothelial cells and human dermal fibroblasts. Biol Cell 2007;99(4):197-207.

[57] Wong VW et al. Surgical approaches to create murine models of human wound healing. J Biomed Biotechnol 2011;2011:969618.

[58] Stephens FO, Dunphy JE, Hunt TK. Effect of delayed administration of corticosteroids on wound contraction. Ann Surg 1971;173(2):214-8.

[59] Clausen OP, Kirkhus B, Schjolberg AR. Cell cycle progression kinetics of regenerating mouse epidermal cells: an in vivo study combining DNA flow cytometry, cell sorting, and $[3 \mathrm{H}] \mathrm{dTh}$ autoradiography. J Invest Dermatol 1986;86(4):402-5.

[60] Hendrickx B et al. Integration of blood outgrowth endothelial cells in derma fibroblast sheets promotes full thickness wound healing. Stem Cells 2010;28(7):1165-77.
[61] Tonello $\mathrm{C}$ et al. In vitro reconstruction of an endothelialized skin substitute provided with a microcapillary network using biopolymer scaffolds. FASEB ] $2005 ; 19(11): 1546-8$

[62] $\mathrm{Wu} \mathrm{X}$ et al. Tissue-engineered microvessels on three-dimensional biodegradable scaffolds using human endothelial progenitor cells. Am Physiol Heart Circ Physiol 2004;287(2):H480-7.

[63] Chen X et al. Prevascularization of a fibrin-based tissue construct accelerates the formation of functional anastomosis with host vasculature. Tissue Eng Part A 2009;15(6):1363-71.

[64] Asakawa $\mathrm{N}$ et al. Pre-vascularization of in vitro three-dimensional tissues created by cell sheet engineering. Biomaterials 2010;31(14): 3903-9.

[65] Tsuda Y et al. Cellular control of tissue architectures using a three-dimensional tissue fabrication technique. Biomaterials 2007;28(33):4939-46.

[66] Sasagawa T et al. Design of prevascularized three-dimensional cell-dense tissues using a cell sheet stacking manipulation technology. Biomaterials 2010;31(7):1646-54

[67] Sorrell JM, Baber MA, Caplan AI. A self-assembled fibroblast-endothelial cell co-culture system that supports in vitro vasculogenesis by both human umbilical vein endothelial cells and human dermal microvascular endothelial cells. Cells Tissues Organs 2007;186(3):157-68.

[68] Kobayashi $\mathrm{H}$ et al. Fibroblast sheets co-cultured with endothelial progenitor cells improve cardiac function of infarcted hearts. J Artif Organs 2008;11(3):141-7.

[69] Sekiya S et al. Three-dimensional cell-dense constructs containing endothelial cell-networks are an effective tool for in vivo and in vitro vascular biology research. Microvasc Res 2010;80(3):549-51.

[70] Black AF et al. In vitro reconstruction of a human capillary-like network in a tissue-engineered skin equivalent. FASEB J 1998;12(13):1331-40.

[71] Gnecchi $\mathrm{M}$ et al. Paracrine mechanisms in adult stem cell signaling and therapy. Circ Res 2008;103(11):1204-19. 